\title{
Altered small-world properties of gray matter networks in breast cancer
}

\author{
S M Hadi Hosseini ${ }^{1}$, Della Koovakkattu ${ }^{1}$ and Shelli R Kesler ${ }^{1,2^{*}}$
}

\begin{abstract}
Background: Breast cancer survivors, particularly those treated with chemotherapy, are at significantly increased risk for long-term cognitive and neurobiologic impairments. These deficits tend to involve skills that are subserved by distributed brain networks. Additionally, neuroimaging studies have shown a diffuse pattern of brain structure changes in chemotherapy-treated breast cancer survivors that might impact large-scale brain networks.

Methods: We therefore applied graph theoretical analysis to compare the gray matter structural networks of female breast cancer survivors with a history of chemotherapy treatment and healthy age and education matched female controls.

Results: Results revealed reduced clustering coefficient and small-world index in the brain network of the breast cancer patients across a range of network densities. In addition, the network of the breast cancer group had less highly interactive nodes and reduced degree/centrality in the frontotemporal regions compared to controls, which may help explain the common impairments of memory and executive functioning among these patients.

Conclusions: These results suggest that breast cancer and chemotherapy may decrease regional connectivity as well as global network organization and integration, reducing efficiency of the network. To our knowledge, this is the first report of altered large-scale brain networks associated with breast cancer and chemotherapy.
\end{abstract}

\section{Background}

Breast cancer is one of the most frequently diagnosed cancers and the leading cause of cancer death among females, accounting for $23 \%$ of the total cancer cases [1]. In the last decade, several neuropsychological studies have shown the negative influence of breast cancer and chemotherapy on various cognitive skills with executive function and memory impairments being the most common $[2,3]$. These deficits have been reported both prior and following chemotherapy with evidence showing increased and/or more severe cognitive changes in breast cancer patients treated with chemotherapy [4-9]. Neuroimaging studies corroborate these findings by showing changes in both brain structure and function associated with breast cancer and chemotherapy [10]. However, it is currently unknown whether breast cancer and chemotherapy affect large-scale brain networks.

\footnotetext{
* Correspondence: skesler@stanford.edu

${ }^{1}$ Department of Psychiatry and Behavioral Sciences, Stanford University School of Medicine, 401 Quarry Road, MC5795 Stanford, CA 94305-5795, USA ${ }^{2}$ Stanford Cancer Center, Palo Alto, CA 94304, USA
}

There are several lines of evidence suggesting that breast cancer may negatively impact whole brain networks. First, while the mechanisms by which breast cancer and its treatments affect cognitive function are largely unknown, possible candidates include neurotoxic effects of chemotherapy, oxidative damage, cytokine dysregulation and individual variation in genes related to neural repair and/or plasticity $[2,3,11,12]$. These candidate mechanisms are likely to have diffuse effects on brain structure. Second, neuroimaging studies indicate that breast cancer survivors show altered brain structure, which would disrupt large-scale networks [13]. Specifically, these patients demonstrate reduced gray matter in bilateral frontal, temporal, cerebellar, thalamic, and cingulate regions as well as decreased white matter integrity in corpus callosum, frontal, and temporal white matter tracts [14-17]. Third, the specific cognitive domains that tend to be most commonly impaired in breast cancer involve executive functions and memory, as noted above. These skills are known to be subserved by distributed, integrated neural networks [18]. Finally, cognitive impairment following breast cancer often tends to be quite

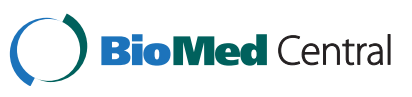


subtle [2], potentially suggesting a more diffuse brain injury. In the current study, we investigated whether breast cancer and chemotherapy are associated with alterations in large-scale structural brain networks.

Recent graph-theoretical analyses have consistently shown that brain structural networks in healthy individuals have small-world characteristics [19]; an architecture that has dense local clustering of connections between neighboring nodes with short path length between any pair of nodes due to the existence of relatively few long-range connections [20]. These characteristics, shared by various biological systems, reflect a network that is simultaneously highly segregated and integrated and allows for higher, more efficient rates of information processing and learning than random networks [21].

Since small-world characteristics were described quantitatively for brain structural networks, there have been multiple graph-theoretical studies seeking to assess the structural correlation networks constructed from regional gray matter volume, cortical thickness and surface area $[12,20,22,23]$. The unique feature of graph-theoretical analysis, compared with the more traditional univariate neuroimaging approaches, is that it can directly test the differences in topological parameters of the brain network such as small-worldness, highly connected hubs and regional network parameters. Whereas univariate neuroimaging approaches have typically shown limited correlations with cognitive function and dysfunction, network parameters may provide a more robust model of cognitive status [24,25]. Recent graph-theoretical studies have illustrated an alteration of arrangements in structural correlation networks associated with normal aging, multiple sclerosis, Alzheimer's disease, schizophrenia and epilepsy [26-30].

In the present study, we applied graph theoretical analyses to compare magnetic resonance imaging (MRI)based gray matter correlation networks of female breast cancer patients treated with chemotherapy and female healthy controls. Considering the lines of evidence regarding a diffuse pattern of gray matter atrophy in breast cancer patients, we hypothesized that such alterations should be reflected in small-world characteristics of the brain structural correlation network. We also examined the between group differences in highly connected hubs as well as in regional network measures such as node betweenness and degree.

\section{Methods \\ Participants}

Thirty-seven female primary breast cancer survivors (BC) age 43-67 years who were treated with systemic chemotherapy (mean time since treatment $=4.5 \pm 3.4$ years) and 38 age-matched healthy controls (CON) participated in the study. BC survivors were recruited via the Army of Women (http://www.armyofwomen.org/), community-based BC support groups and local media advertisements. Healthy controls were recruited via the Army of Women and local media advertisements. Groups were matched for age, education, premorbid cognitive functioning and minority status although there were significantly more post-menopausal women in the $\mathrm{BC}$ group (Table 1). Premorbid cognitive functioning was measured using the Information subtest of the Wechsler Adult Intelligence Scale 4th Edition. This subtest measures fund of general knowledge and is believed to be influenced by previous experience and quality of education, making it a good estimate of premorbid ability [31]. Participants in the BC group were free from disease and had no history of relapse or recurrence at the time of evaluation. Chemotherapy regimens included AC (Doxorubicin, Cyclophosphamide) $(\mathrm{N}=9), \quad$ ACT (Doxorubicin, Cyclophosphamide, Paclitaxel) $(\mathrm{N}=16)$, ACF (Doxorubicin, Cyclophosphamide, 5-Fluorouracil) $(\mathrm{N}=2)$, CT (Cyclophosphamide, Paclitaxel) $(\mathrm{N}=6), \mathrm{CMF}$ (Cyclophosphamide, Methotrexate, 5-Fluorouracil) $(\mathrm{N}=2)$, CTF (Cyclophosphamide, Paclitaxel, 5-Fluorouracil) $(\mathrm{N}=1)$ and $\mathrm{AC}+\mathrm{CMF}(\mathrm{N}=1)$. Additionally, 15 women were treated with tamoxifen and 25 received radiation therapy. None of the BC patients had cerebral metastasis or had been treated for that. Participants were excluded for neurologic, psychiatric or medical conditions known to affect cognitive function (e.g. learning disability, traumatic brain injury, chronic depression) as well as any MRI contraindications (e.g. non-MRI safe implants). This study was approved by the Stanford

Table 1 Demographic data for the breast cancer and healthy control groups

\begin{tabular}{|c|c|c|c|c|}
\hline & $B C(N=37)$ & $\operatorname{CON}(\mathrm{N}=38)$ & t or Chi Sq. & p-value \\
\hline age & $54.2(6.1)$ & $55.5(9.0)$ & .737 & .46 \\
\hline education & $16(2.8)$ & $17(2.6)$ & 1.61 & .11 \\
\hline minority status & $8.6 \%$ & $10.5 \%$ & 2.27 & .69 \\
\hline post menopause & $86.7 \%$ & $55.9 \%$ & 7.24 & .007 \\
\hline premorbid cognitive status* & $13.0(6.0)$ & $13.8(2.7)$ & .774 & .44 \\
\hline
\end{tabular}

${ }^{*}$ measured using the Information subtest of the Wechsler Adult Intelligence Scale 4th Edition.

Data are shown as mean (standard deviation) except where noted. 
University Institutional Review Board and all participants provided informed consent.

\section{MRI data acquisition and preprocessing}

MRI scanning was performed on a GE Discovery MR750 3.0 Tesla whole body scanner (GE Medical Systems, Milwaukee, WI). High-resolution T1-weighted images were acquired with 3D spoiled gradient echo pulse sequence using the following parameters: $\mathrm{TR}=8.5 \mathrm{~ms}, \mathrm{TE}=3.396$, $\mathrm{TI}=400 \mathrm{~ms}$, flip angle $=15, \mathrm{FOV}=220 \mathrm{~mm}$, number of excitation $=1$, acquisition matrix $=256 \times 192$. Totally, 124 contiguous coronal slices were with in-plane resolution of $0.859 \mathrm{~mm} \times 0.859 \mathrm{~mm}$.

Image preprocessing was performed using Statistical Parametric Mapping 8 (SPM8; Wellcome Department of Cognitive Neurology, London, UK; http://www.fil.ion. ucl.ac.uk/spm/). The images were initially segmented into gray matter (GM), white matter, and cerebrospinal fluid images based on the ICBM Tissue Probabilistic Maps (http://www.loni.ucla.edu/ICBM/ICBM TissueProb.html). A study-specific a priori probability map of GM was created from the modulated spatially normalized segmented GM images using the Template-O-Matic (TOM8) toolbox [32]. Next, the custom priors were affine-registered to the standard Montreal Neurological Institute (MNI) space.

Voxel-based morphometry (VBM) preprocessing steps were performed using the VBM8 toolbox for SPM8 (http://dbm.neuro.uni-jena.de/vbm/) and this involved segmentation of MR images into GM tissue segments using the standard unified segmented and non-linearly warping of the tissue segments to the GM study-specific customized template model [33]. Images were then modulated to ensure that relative volumes of GM were preserved following the spatial normalization procedure. Sample homogeneity was checked to identify any outliers in the study population. Data for one participant in the $\mathrm{BC}$ group were excluded from the analysis because of a covariance below $2 \mathrm{SD}$ and confirmation of visual motion artifact.

\section{ROI extraction}

There are different nodal definition methods in brain network analysis. While the results might be affected by the choice of parcellation scheme, recent evidence showed that the results of between-group comparison remain intact regardless of the applied parcellation scheme [34]. We generated 90 cortical and subcortical regions of interest (ROIs), excluding the cerebellum, from the Automated Anatomical Labeling (AAL) atlas using the WFU PickAtlas Toolbox [35]. The ROIs were identical to those used in a previous graph analysis study of typical brain development by Fan and colleagues [22]. These AAL ROIs were resliced to the same dimension as that of tissue segmented images obtained from the VBM preprocessing step. The ROIs were subsequently used to mask the individual modulated, normalized GM images and extract the average volume within each ROI using the REX toolbox (http://web.mit.edu/swg/software. htm). A linear regression analysis was performed at every ROI to remove the effects of age and total brain volume. The residuals of this regression were then substituted for the raw ROI volume values $[22,27,36]$.

\section{Construction of structural correlation network}

The extracted residual volumes of all 90 anatomical ROIs were used for construction of structural correlation networks. For each group, a $90 \times 90$ association matrix $R$ was generated with each entry $r_{i j}$ defined as the Pearson correlation coefficient between residual volumes of regions $i$ and $j$, across subjects. From each association matrix, a binary adjacency matrix $A$ was derived where $a_{i j}$ was considered 1 if $r_{i j}$ was greater than a specific threshold and zero otherwise. The diagonal elements of the constructed association matrix were also set to zero. The resultant adjacency matrix represented a binary undirected graph $G$ in which regions $i$ and $j$ were connected if $g_{i j}$ was unity. Therefore, a graph was constructed with $N=90$ nodes (anatomical ROIs), with a network degree of $E$ equal to number of edges (links), and a network density (cost) of $\mathrm{D}=\mathrm{E} /[(\mathrm{N} \times(\mathrm{N}-1)) / 2]$ representing the fraction of present connections to all possible connections. Since thresholding the association matrices of different groups at an absolute threshold results in networks with a different number of nodes (and degrees) that might influence the network measures and reduce interpretation of between group results [37], two approaches were implemented for thresholding the constructed association matrices based on previous studies [26-28]: (1) Thresholding the constructed association matrices at a minimum network density in which all nodes become fully connected in the brain networks of both groups; (2) Thresholding the constructed association matrices at a range of network densities and comparing the network topologies across that range.

\section{Network analysis}

Small-worldness The small-worldness of a complex network, as described above, has two key metrics: the clustering coefficient $C$ and the characteristic path length $L$ of the network. The clustering coefficient of a node is a measure of the number of edges that exist between its nearest neighbors. The clustering coefficient of a network is thus the average of clustering coefficients across nodes and is a measure of network segregation. The characteristic path length of a network is the average shortest path length between all pairs of nodes in 
the network and is the most commonly used measure of network integration [38]. To evaluate the topology of the brain network, these parameters must be compared to the corresponding mean values of a random graph with the same number of nodes, total edges, and degree distribution as the network of interest $[39,40]$. Thus, we obtained the small-worldness index of a network as $\left[\mathrm{C} / \mathrm{C}_{\text {rand }}\right] /\left[\mathrm{L} / \mathrm{L}_{\text {rand }}\right]$ where $C_{\text {rand }}$ and $L_{\text {rand }}$ are the mean clustering coefficient and the characteristic path length of the random network [20]. In a small-world network, the clustering coefficient is significantly higher than that of random networks $\left(C / C_{\text {rand }}\right.$ ratio greater than 1$)$ while the characteristic path length is comparable to random networks $\left(L / L_{\text {rand }}\right.$ ratio close to 1$)$.

Regional network measures We also investigated the nodal characteristics of the constructed structural networks to identify differences in regional network measures between groups. Nodal betweenness centrality and nodal degree were calculated for each of the anatomical ROIs for the networks thresholded at minimum density with full-connectivity. Nodal betweenness centrality is defined as the fraction of all shortest paths in the network that pass through a given node and is used to detect important anatomical or functional connections. Nodes that bridge disparate parts of the network have a high betweenness centrality [38]. On the other hand, nodal degree is defined as the number of connections that a node has with the rest of the network and is considered a measure of interaction of a node, structurally or functionally, with the network.

Network hubs Hubs are crucial components for efficient communication in a network. Hubs are not only considered as important regulators of information flow but also play a key role in network resilience to insult [38]. We considered a node as a hub if its betweenness centrality was at least 2SD higher than mean network centrality [26].

Comparing correlation strengths between groups To test the significance of differences in overall interregional correlation of gray matter volume between networks, the correlation coefficients were converted to $z$ values using Fisher's r-to-z transform. This transformation resulted in values that were approximately normally distributed [28]. Then, a two sample $t$-test was used to test the significance of the difference in mean overall correlation between groups.

Comparing network measures between groups In order to test the statistical significance of the betweengroup differences in network topology and regional network measures, a non-parametric permutation test with
1000 repetitions was used [26,28]. In each repetition, the calculated residual volumes of each participant were randomly reassigned to one of the two groups so that each randomized group had the same number of subjects as the original groups. Then, an association matrix was obtained for each randomized group. The binary adjacency matrices were then estimated by thresholding the association matrices at a range of network densities. The network measures were then calculated for all the networks at each density. The differences in network measures between randomized groups were then calculated resulting in a permutation distribution of difference under the null hypothesis. The actual between-group difference in network measures was then placed in the corresponding permutation distribution and a two-tailed p-value was calculated based on its percentile position [27].

We used the Brain Connectivity Toolbox [38] for quantification of network measures in addition to inhouse software, Graph Analysis Toolbox (http://nnl. stanford.edu/Tools.html), for comparing the structural networks. Brain Net Viewer (http://www.nitrc.org/ projects/bnv/) was used for visualization of the graphs.

It should be noted that we could not statistically explore the relationship between cognitive-behavioral measures and the extracted network properties. It is because the extracted network measures are group-specific and can not be quantified for individuals. Therefore, we could not perform statistical analysis (e.g. correlation analysis) to relate network properties and cognitivebehavioral outcomes.

\section{Results}

Comparing networks at minimum density with full-connectivity

The minimum network density in which all nodes became fully connected in the structural networks of both groups was 0.184 . The association matrices for each group and corresponding binary adjacency matrices thresholded at the minimum density are shown in Figure 1. The correlation network of the $\mathrm{BC}$ group showed lower overall correlation strength than the CON group $(t=5.9, P<0.001)$. Topological measures for the networks of both groups thresholded at the density of 0.184 are given in Table 2 . In both groups, the normalized characteristic path length of the network was close to 1 and the normalized clustering coefficient was greater than 1 which results in a small-worldness parameter of greater than 1 . Thus, consistent with previous studies, the gray-matter correlation network of both groups followed a small-world property. While no significant difference in characteristic path length was observed between networks $(P=0.22)$, the normalized 


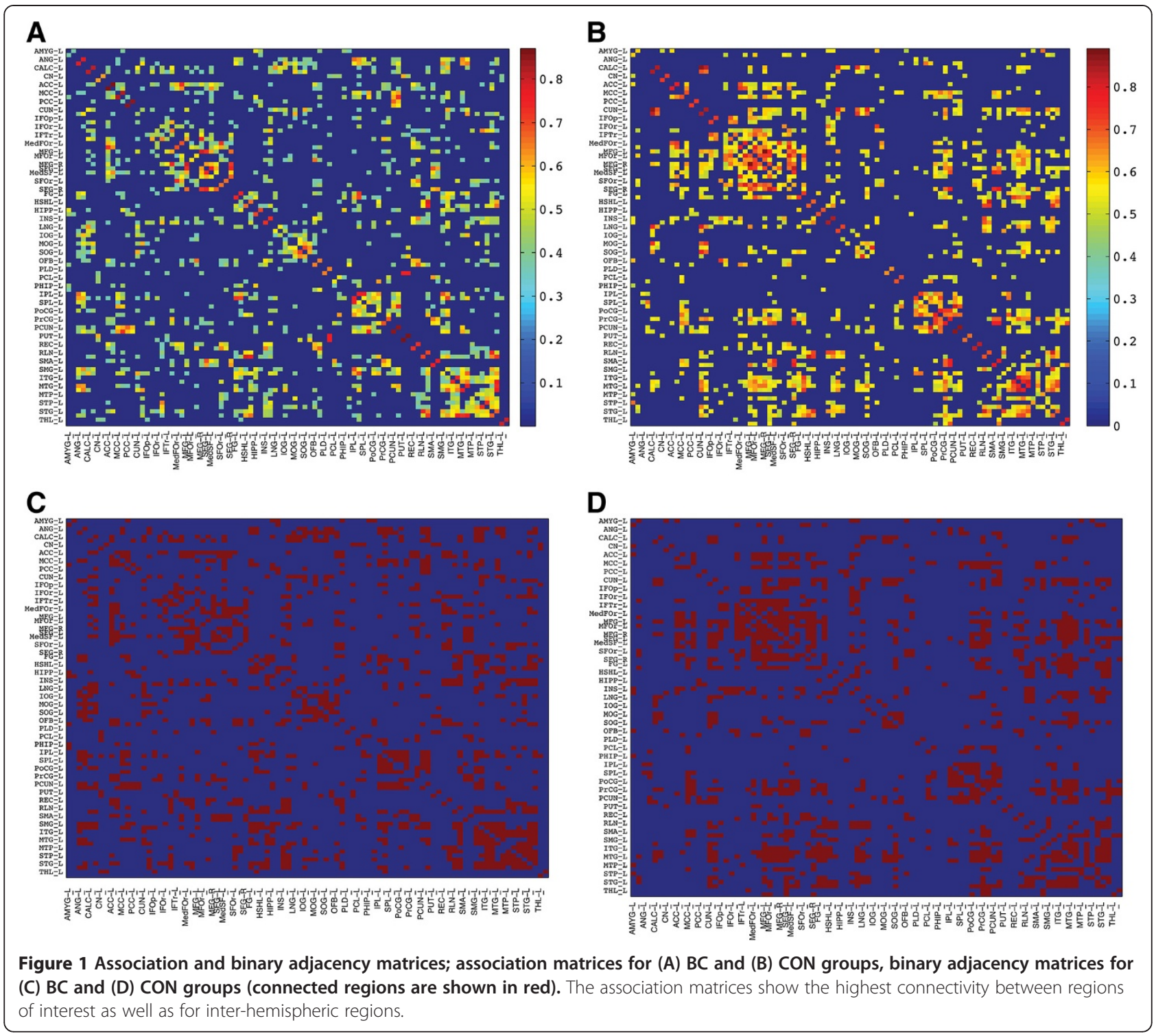

clustering coefficient was significantly higher in CON network $(P<0.05)$. Consequently, the small-worldness parameter was found to be greater in the CON network than in the $\mathrm{BC}$ network and the observed difference was marginally significant $(P=0.08)$.

Table 2 Network measures at minimum density of $\mathbf{0 . 1 8 4}$ with full connectivity

\begin{tabular}{llll}
\hline & BC & CON & p-value \\
\hline Density & 0.184 & 0.184 & - \\
\hline Mean clustering coefficient & 0.485 & 0.517 & 0.16 \\
\hline Characteristic path length & 2.11 & 2.2 & 0.23 \\
\hline Normalized clustering & 2.65 & 3.06 & 0.03 \\
\hline Normalized path length & 1.15 & 1.20 & 0.22 \\
\hline Small-worldness & 2.30 & 2.54 & 0.08 \\
\hline
\end{tabular}

\section{Comparing networks across a range of density}

In order to investigate changes in the network topology as a function of network density (cost), we thresholded the constructed association matrices at a range of network densities. Changes in network parameters are depicted in Figure 2. The figures show that both networks follow a small-world organization across the range of densities. The results of comparison of the two networks as a function of network density are shown in Figure 3. While there was no significant difference in network characteristic path lengths between groups (same level of integration), the clustering coefficient was significantly higher (more segregated network) in the CON network in various densities resulting in higher small-worldness compared to the $\mathrm{BC}$ group across a range of densities. 


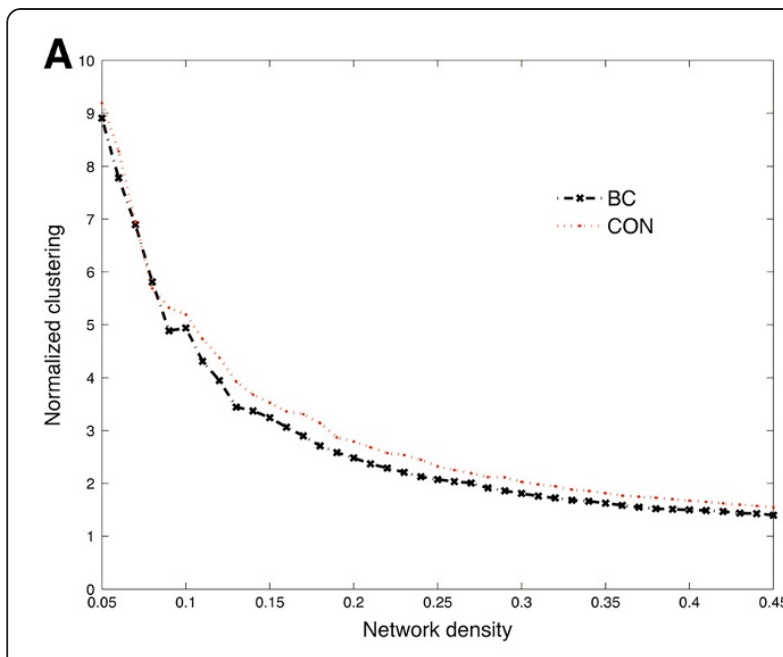

B

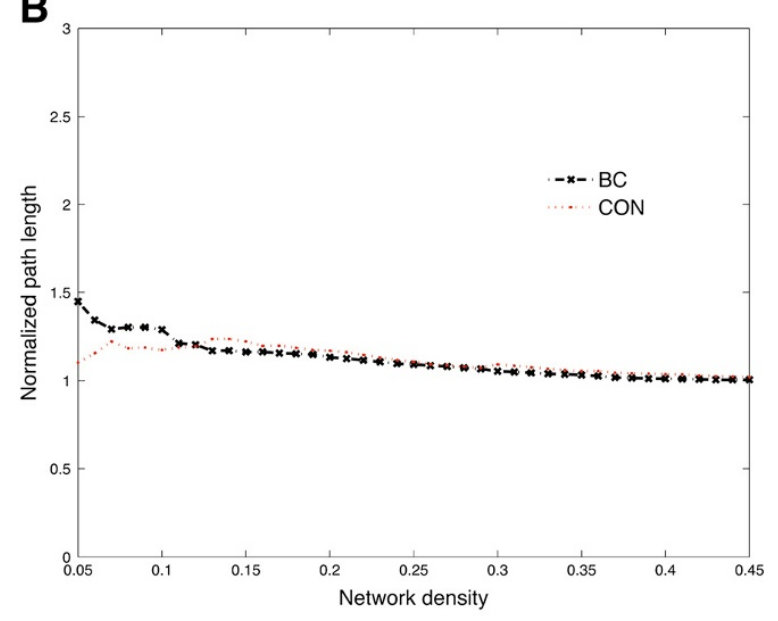

C

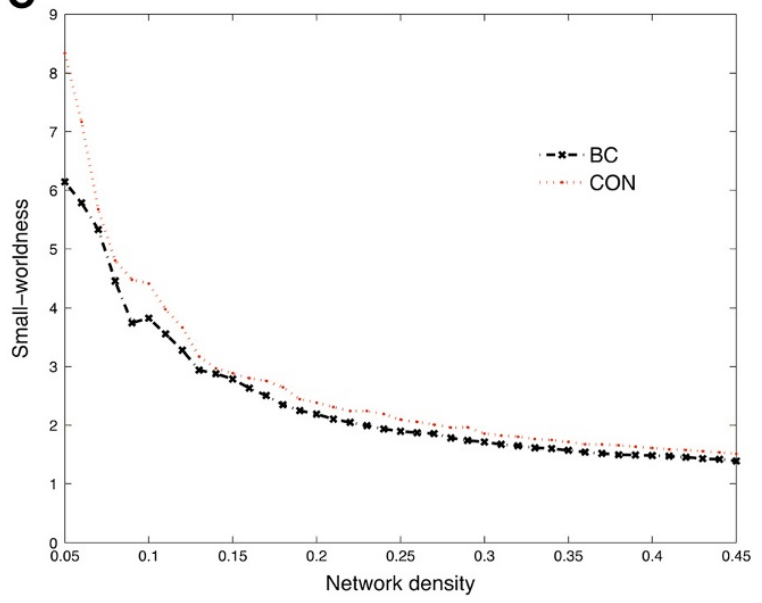

Figure 2 Global network measures; A) clustering coefficient, B) characteristic path length and C) small-worldness of $B C$ and CON networks. The figures show that both networks follow a

small-world organization across the range of densities; the characteristic path length is close to 1 (for the fully connected network, i.e.

density $>0.19$ ) and the clustering is greater than 1 in different densities.
Below the minimum density of full connectivity (0.184), the BC network fragments sooner that the CON network and thus the normalized path length of the $\mathrm{BC}$ network increases relatively faster compared with that of CON network. This results in a relatively large drop in the difference in normalized path length between groups in densities below 0.184 .

\section{Regional network measures}

As shown in Figure 4, the CON group demonstrated higher nodal betweeness/degree in several frontal and temporal regions. The $\mathrm{BC}$ group demonstrated higher nodal betweeness/degree primarily in parieto-occipital regions but also in the anterior cingulate and left thalamus.

\section{Network hubs}

In the $\mathrm{BC}$ group, network hubs were identified at left anterior cingulate, right inferior parietal lobule, and right supramarginal gyrus. In CON group, hubs were found in right middle frontal gyrus, left superior frontal gyrus, right insula, right precuneus, and left middle temporal gyrus (Figure 5).

\section{Discussion}

We applied graph theoretical analyses to compare gray matter volume correlation networks of female primary breast cancer $(\mathrm{BC})$ survivors treated with chemotherapy and female healthy controls $(\mathrm{CON})$. We found an alteration in small-world characteristics of the brain structural network in the $\mathrm{BC}$ group; an observation that confirms our hypothesis suggesting changes in largescale brain network properties in BC survivors treated with chemotherapy. To our knowledge, this is the first report of altered large-scale network properties associated with $\mathrm{BC}$ and chemotherapy.

\section{Between-group differences in network topology}

While the structural correlation networks of both groups followed a small-world organization, the results revealed a decrease in the small-world index of the $\mathrm{BC}$ group compared to controls. The characteristic path lengths of the $\mathrm{BC}$ and $\mathrm{CON}$ group networks were not different whereas the clustering coefficient was significantly smaller in the $\mathrm{BC}$ network than in the CON network. Decreased clustering coefficient and small-world index in the $\mathrm{BC}$ group was observed not only at the minimum network density but also over a range of density thresholds.

These findings suggest that the structural correlation networks of $\mathrm{BC}$ patients tend to have a more randomized configuration compared to $\mathrm{CON}$ group associated with weaker regional connectivity and disrupted global organization [38]. Given that $\mathrm{BC}$ tends to be a disease 

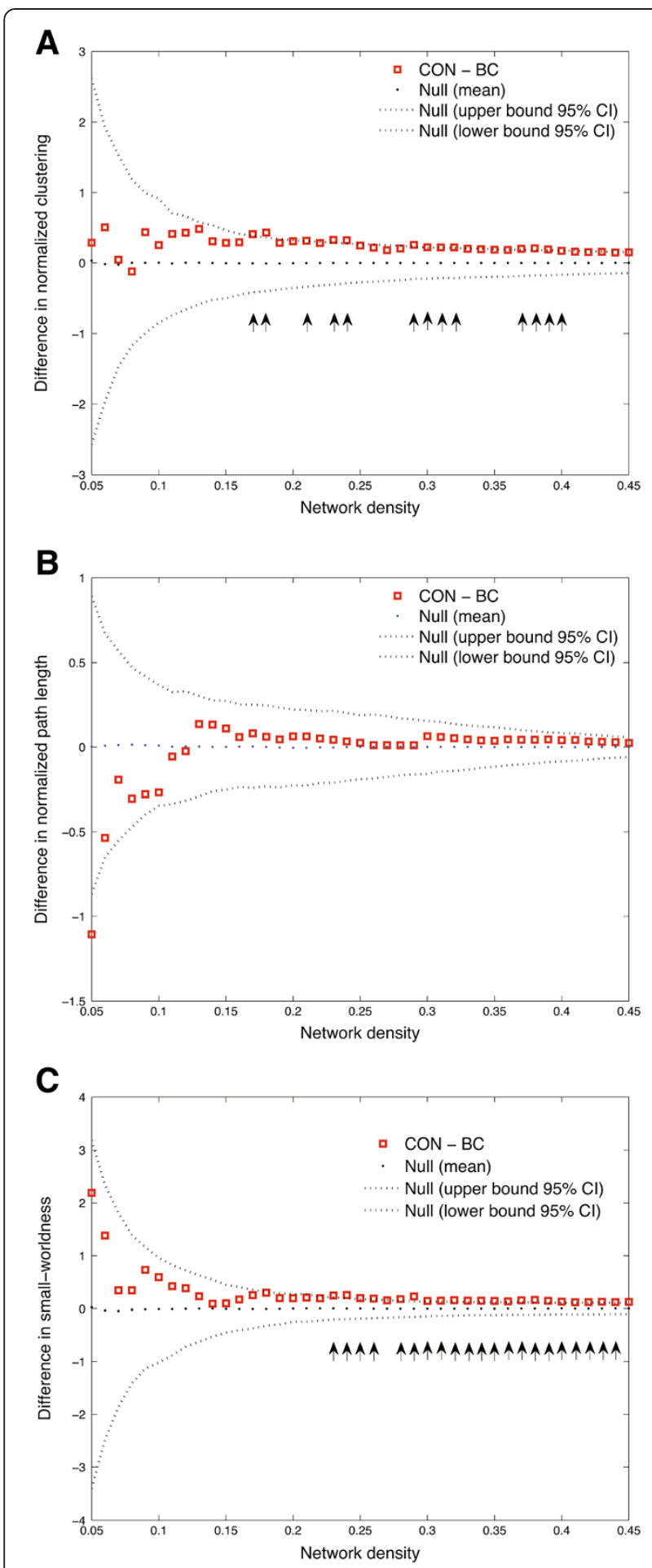

Figure 3

associated with aging, altered network topology could make women with $\mathrm{BC}$ more vulnerable to age-related cognitive decline. Recent studies suggest that older BC patients show increased chemotherapy-related cognitive deficits [4,5]. Additionally, this same profile of network
Figure 3 Between-group differences in global network measures; between-group differences in A) clustering coefficient, B) characteristic path length and C) small-worldness parameter. The vertical arrows represent the densities where the difference is statistically significant at $P<0.05$. It shows that while there is no significant difference in network characteristic path lengths between groups (same level of integration), the clustering coefficient is significantly higher (more segregated network) in the CON network in various densities resulting in higher small-worldness compared to the BC group across a range of densities. Squares represent control minus BC group measure, dashed lines show the 95\% confidence interval and the dotted line shows the mean for the random graph distribution.

topology (i.e. decreased clustering and small-world index) has been associated with Alzheimer's dementia [41]. The interface between aging, breast cancer and cognitive decline will be a critical area of continued investigation.

The observed lack of densely interconnected neighbors in the network of $\mathrm{BC}$ group is also supported by the lower overall correlation strength in the association matrix of $\mathrm{BC}$ group compared to CON group. Together, the results of network-level analysis corroborates previous structural neuroimaging findings that have demonstrated a diffuse pattern of atrophy in gray matter volumes in BC patients $[15,16]$ and further suggests an alteration in the coordinated patterns of brain morphology in the structural network of these patients.

One potential mechanism underlying this networklevel alteration is white matter damage. There is a large body of evidence suggesting that global gray matter atrophy is associated with focal and global white matter damage due in part to the transection of axons and subsequent retrograde neuronal loss [42,43]. Animal studies show that chemotherapy suppresses neural progenitor cell proliferation responsible for white-matter tract integrity and cortico-cortical connections [44-48]. Thus, the observed network-level alteration in structural correlation network of $\mathrm{BC}$ patients might arise from neurotoxic effects of chemotherapy on cortico-cortical connections. This idea is also supported by a recent diffusion weighted imaging study involving chemotherapy-treated $\mathrm{BC}$ survivors that reported a diffuse pattern of microstructural white matter damage [14].

\section{Between-group differences in regional network measures} High degree/betweenness nodes were observed mainly in frontotemporal regions in the CON network and in parietal, cingulate, and occipital regions in the $\mathrm{BC}$ network. Nodes with high degree/centrality in the structural network identify regions that are highly interactive and have the potential to participate in a large number of functional interactions [49]. The results indicate that the 


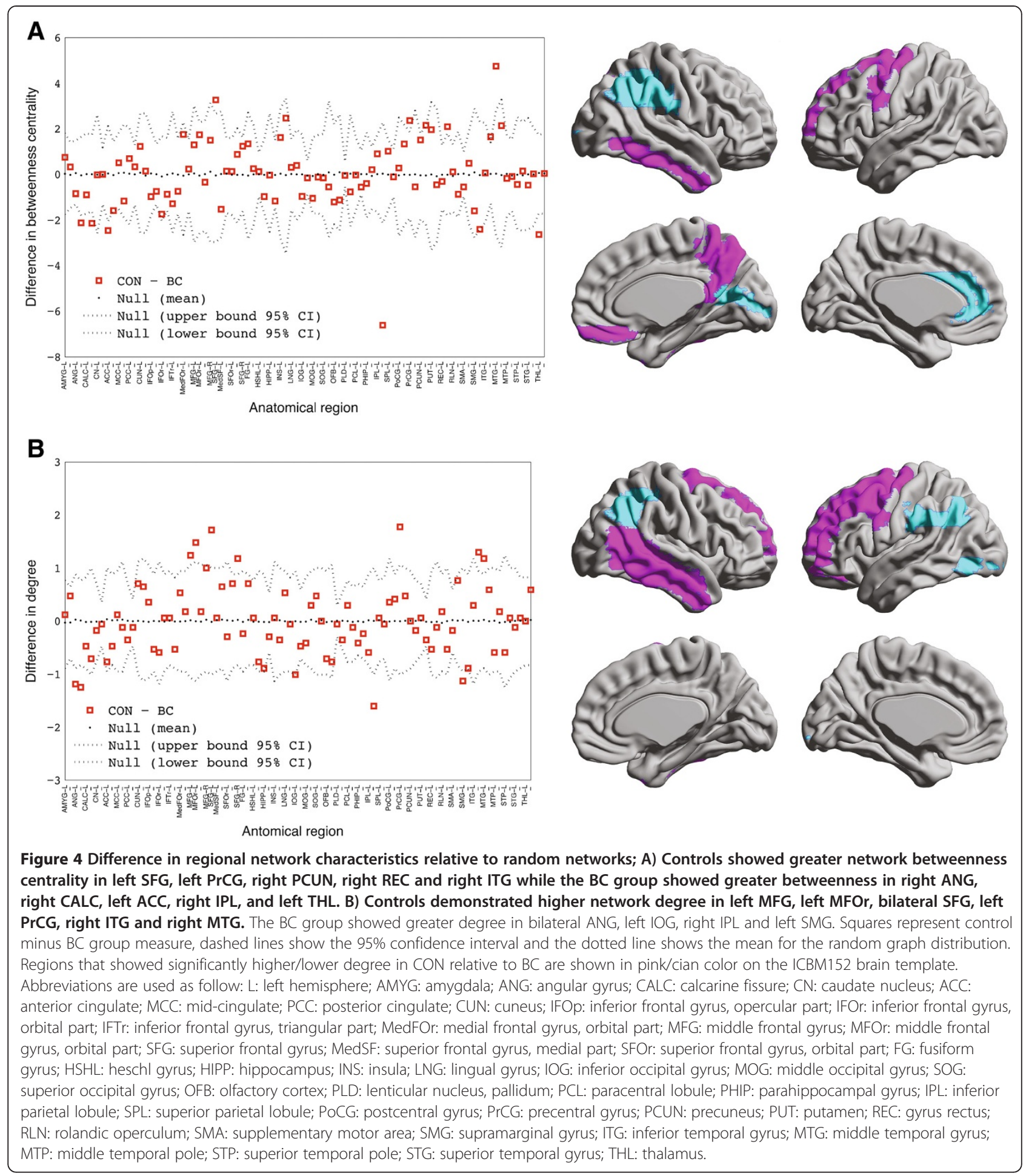

network of BC group has less highly-interactive nodes in the frontotemporal regions.

Reduction in gray and white matter volumes in frontotemporal regions of patients with $\mathrm{BC}$ before and after treatment has been shown in a recent study [15]. A number of functional neuroimaging studies have also reported increased activation in the frontotemporal network in these patients suggesting necessity of recruiting a compensatory neural mechanism to complete certain tasks, or decreased efficiency of these systems [10,50,51]. The observed changes in the centrality of the frontotemporal nodes in the network of BC patients is consistent 

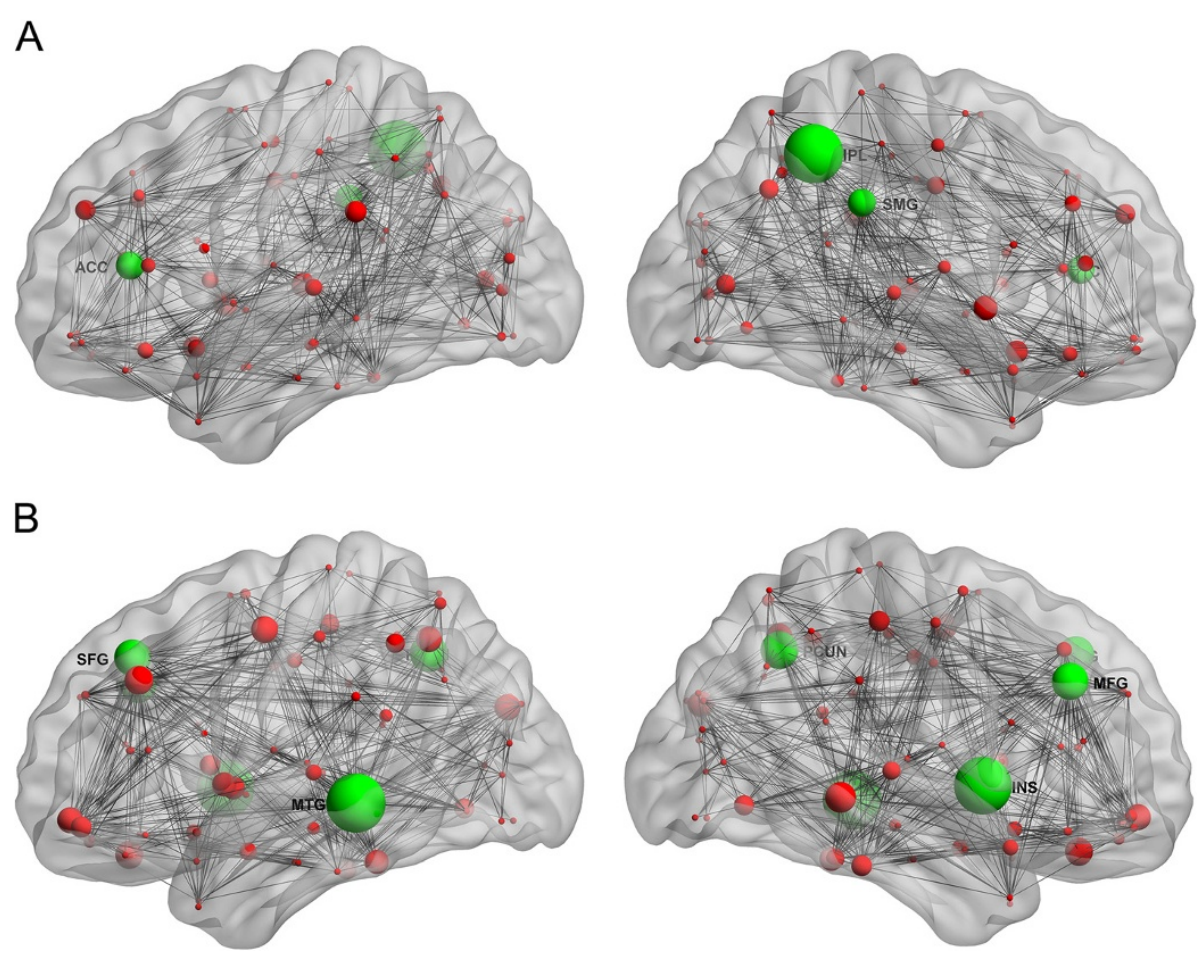

Figure 5 Network hubs; structural correlation networks and hubs overlaid on ICBM152 brain template for A) BC and B) CON groups. Grey lines indicate connections and spheres represent regions. The radius of the spheres is proportional to the nodal betweenness. Hubs are shown in green color.

with these previous results and may explain the impairment of these patients in memory and executive functioning.

There are several mechanisms that could explain the observed change in the distribution of highly influential nodes in BC group with less highly-interactive nodes in the prefrontal and temporal cortices. Certain genetic and immunologic factors have been proposed as important contributors to cognitive decline in patients with $\mathrm{BC}$ [3]. For example, apolipoprotein E (APOE) is a complex protein that has an important role in neuronal repair and plasticity after injury [52]. The relationship between this genotype and adverse cognitive performance and reduce mesial temporal (hippocampal) volume has been shown in long-term survivors of $\mathrm{BC}$ [53]. Also, cytokines that become dysregulated during cancer and/or chemotherapy treatment have been shown to affect cognitive function in healthy adults [54]. Cytokines have important roles in central nervous system functioning including modulation of dopamine and other neurotransmitters [55] that affect the frontotemporal network [56]. The effects of genotypic variations and cytokine regulation on large-scale networks have not been established and require further investigation.

The BC group had significantly more post-menopausal women than the healthy comparison group. This is expected given that chemotherapy frequently causes early menopause [57] and tamoxifen can increase menopausal symptoms [19], both by blocking or reducing estrogen. Estrogen has a marked effect on brain function, particularly in frontotemporal regions and their concomitant executive and memory functions [58]. Previous studies suggest that tamoxifen, in addition to chemotherapy, can have detrimental effects on cognitive and neurobiologic outcomes in BC survivors $[59,60]$. The present findings may indicate potential secondary neuroendocrine effects on large-scale brain networks.

\section{Network hub analysis}

There was a difference in the number and distribution of hubs between the $\mathrm{BC}$ and $\mathrm{CON}$ groups. We found six network hubs in the CON group mainly across frontal and temporal regions compared to three hubs in the $\mathrm{BC}$ group primarily in parietal and cingulate areas. The identified hubs in the CON group are consistent with the results of previous graph-theoretical analysis involving healthy adults $[26,28]$. The present findings indicate that the network of $\mathrm{BC}$ patients does not show expected prefrontal and temporal hubs suggesting network alterations involving these regions, again which are critical for the executive function and memory skills commonly disrupted in $\mathrm{BC}$. 
Additionally, the number of identified hubs in the $\mathrm{BC}$ network was half the number of hubs in the CON network. Since hubs are crucial components for efficient communication across the network, this observation suggests a less efficient information transfer in the network of $\mathrm{BC}$ patients. These results are consistent with our previous findings showing that $\mathrm{BC}$ survivors can often perform similarly to controls on cognitive tasks but require significantly more neural resources to do so [50]. Standardized neurocognitive tests may lack sensitivity to these subtle changes [2] as most were designed to assess specific cognitive skills. Based on the present findings, measures of reaction time or parallel processing should be included in evaluations of BC-related cognitive outcome. Quantitative process measures might also be more effective as these assessments examine the efficiency of the patient's performance strategies in addition to performance accuracy [61].

This study has several limitations. First, this was a cross-sectional study and therefore, we could not directly test the dissociated effects of cancer and chemotherapy on network measures. Future studies can address this issue through longitudinal evaluations of network measures in patients with BC. In addition, future studies might investigate whether the alteration in gray matter network is due to any cancer (and not just breast cancer). It is also important to dissociate between cancer-related physiological changes and cancer effects on mental states. Second, the network measures were identified by calculating the correlations of gray matter volume across subjects. While this methodology is common for investigating between-group differences in brain structural network, it lacks the ability to explore the individual differences in network parameters. Specifically, we could not explore the effect of treatment period, regimen, age or other individual differences on network parameters or investigate the effects of altered network parameters on cognitive-behavioral outcome.

\section{Conclusions}

Despite these limitations, these findings increase our understanding of $\mathrm{BC}$ and chemotherapy-related cognitive impairment by demonstrating alterations in specific network properties. Survivors of BC treated with chemotherapy showed decreased regional connectivity (clustering coefficient) and global network organization (smallworldness) and integration (hubs) suggesting reduced robustness and efficiency of the network. A pattern of frontotemporal abnormality was also noted. These results contribute novel insights regarding the neurobiologic mechanisms underlying cognitive deficits in these patients and highlight critical areas for future research related to the potential vulnerability of $\mathrm{BC}$ patients to the neurologic effects of aging and other forms of structural damage.

\section{Competing interests}

None.

\section{Acknowledgements}

This work was supported by the National Institutes of Health Director's New Innovator Award [DP2 OD004445-01 to SK]

\section{Authors' contributions}

$\mathrm{HH}$ wrote code for the analysis toolbox, analyzed the graph theory data and co-wrote the manuscript. DK assisted with volumetric data acquisition and analyses and co-wrote the manuscript. SK conceived and directed development of the analysis toolbox, designed and supervised the study, conducted demographic and behavioral data analyses and

co-wrote the manuscript.

Received: 1 December 2011 Accepted: 28 May 2012

Published: 28 May 2012

\section{References}

1. Jemal A, Bray F, Center MM, Ferlay J, Ward E, Forman D: Global cancer statistics. CA: a cancer journal for clinicians; 2011.

2. Vardy J: Cognitive function in breast cancer survivors. Cancer Treat Res 2009, 151:387-419.

3. Janelsins MC, Kohli S, Mohile SG, Usuki K, Ahles TA, Morrow GR: An update on cancer- and chemotherapy-related cognitive dysfunction: current status. Semin Oncol 2011, 38:431-438.

4. Kesler SR, Kent J, O'Hara R: Prefrontal cortex and executive function impairments in primary breast cancer. Arch Neurol 2011, 68:1447-1453.

5. Ahles TA, Saykin AJ, McDonald BC, Li Y, Furstenberg CT, Hanscom BS, Mulrooney TJ, Schwartz GN, Kaufman PA: Longitudinal Assessment of Cognitive Changes Associated With Adjuvant Treatment for Breast Cancer: Impact of Age and Cognitive Reserve. J Clin Oncol 2010, 28:4434-4440

6. Quesnel C, Savard J, Ivers H: Cognitive impairments associated with breast cancer treatments: results from a longitudinal study. Breast Cancer Res Treat 2009, 116:113-123.

7. Schagen SB, Muller MJ, Boogerd W, Mellenbergh GJ, van Dam FS: Change in cognitive function after chemotherapy: a prospective longitudinal study in breast cancer patients. J Natl Cancer Inst 2006, 98:1742-1745.

8. Jansen CE, Cooper BA: Dodd MJ. Miaskowski CA: A prospective longitudinal study of chemotherapy-induced cognitive changes in breast cancer patients. Support Care Cancer; 2010

9. Wefel JS, Saleeba AK, Buzdar AU, Meyers CA: Acute and late onset cognitive dysfunction associated with chemotherapy in women with breast cancer. Cancer 2010, 116:3348-3356.

10. de Ruiter MB, Reneman L, Boogerd W, Veltman DJ, van Dam FS, Nederveen AJ, Boven E, Schagen SB: Cerebral hyporesponsiveness and cognitive impairment 10 years after chemotherapy for breast cancer. Hum Brain Mapp 2011, 32:1206-1219.

11. Wefel JS, Witgert ME, Meyers CA: Neuropsychological sequelae of noncentral nervous system cancer and cancer therapy. Neuropsychol Rev 2008, 18:121-131.

12. Ahles TA, Saykin AJ: Candidate mechanisms for chemotherapy-induced cognitive changes. Nat Rev Cancer 2007, 7:192-201.

13. Seeley WW, Crawford RK, Zhou J, Miller BL, Greicius MD: Neurodegenerative diseases target large-scale human brain networks. Neuron 2009, 62:42-52.

14. Deprez S, Amant F, Yigit R, Porke K, Verhoeven J, Van den Stock J, Smeets A, Christiaens MR, Leemans A, Van Hecke W, et al: Chemotherapy-induced structural changes in cerebral white matter and its correlation with impaired cognitive functioning in breast cancer patients. Hum Brain Mapp 2011, 32:480-493.

15. McDonald BC, Conroy SK, Ahles TA, West JD, Saykin AJ: Gray matter reduction associated with systemic chemotherapy for breast cancer: a prospective MRI study. Breast Cancer Res Treat 2010 123:819-828.

16. Inagaki M, Yoshikawa E, Matsuoka Y, Sugawara Y, Nakano T, Akechi T, Wada $\mathrm{N}$, Imoto S, Murakami K, Uchitomi Y: Smaller regional volumes of brain 
gray and white matter demonstrated in breast cancer survivors exposed to adjuvant chemotherapy. Cancer 2007, 109:146-156.

17. Abraham J, Haut M, Moran M, Filburn S, Lemiuex S, Kuwabara H: Adjuvant chemotherapy for breast cancer: effects on cerebral white matter seen in diffusion tensor imaging. Clin Breast Cancer 2008, 8:88-91.

18. Leh SE, Petrides M, Strafella AP: The Neural Circuitry of Executive Functions in Healthy Subjects and Parkinson's Disease. Neuropsychopharmacology 2010, 35:70-85.

19. Bakkum-Gamez JN, Laughlin SK, Jensen JR, Akogyeram CO, Pruthi S: Challenges in the Gynecologic Care of Premenopausal Women With Breast Cancer. Mayo Clin Proc 2011, 86:229-240.

20. Bassett DS, Bullmore E: Small-world brain networks. Neuroscientist 2006, 12:512-523.

21. Simard D, Nadeau L: Fastest learning in small-world neural networks. Physics Letters A 2005, 336:8-15.

22. Fan $Y$, Shi F, Smith JK, Lin W, Gilmore JH, Shen D: Brain anatomical networks in early human brain development. Neurolmage 2011, 54:1862-1871

23. Chen ZJ, He Y, Rosa-Neto P, Germann J, Evans AC: Revealing modular architecture of human brain structural networks by using cortical thickness from MRI. Cerebral cortex 2008, 18:2374-2381.

24. Petrella JR: Use of graph theory to evaluate brain networks: a clinical tool for a small world? Radiology 2011, 259:317-320.

25. Sporns O, Chialvo DR, Kaiser M, Hilgetag CC: Organization, development and function of complex brain networks. Trends Cogn Sci 2004, 8:418-425.

26. Bassett DS, Bullmore E, Verchinski BA, Mattay VS, Weinberger DR, MeyerLindenberg A: Hierarchical organization of human cortical networks in health and schizophrenia. J Neurosci 2008, 28:9239-9248.

27. Bernhardt BC, Chen Z, He Y, Evans AC, Bernasconi N: Graph-Theoretical Analysis Reveals Disrupted Small-World Organization of Cortical Thickness Correlation Networks in Temporal Lobe Epilepsy. Cerebral Cortex 2011, 21(9):2147-2157.

28. He Y, Chen Z, Evans A: Structural insights into aberrant topological patterns of large-scale cortical networks in Alzheimer's disease. J Neurosci 2008, 28:4756-4766.

29. Wu K, Taki Y, Sato K, Kinomura S, Goto R, Okada K, Kawashima R, He Y, Evans AC, Fukuda H: Age-related changes in topological organization of structural brain networks in healthy individuals. Hum Brain Mapp 2011, 33 (3):552-568.

30. He Y, Dagher A, Chen Z, Charil A, Zijdenbos A, Worsley K, Evans A: Impaired small-world efficiency in structural cortical networks in multiple sclerosis associated with white matter lesion load. Brain 2009, 132:3366-3379.

31. Lowe DA, Rogers SA: Estimating Premorbid Intelligence among Older Adults: The Utility of the AMNART. J Aging Res 2011, 2011:428132.

32. Wilke M, Holland S, Altaye M, Gaser C: Template-O-Matic: A toolbox for creating customized pediatric templates. Neurolmage 2008, 41:903-913.

33. Ashburner J, Friston KJ: Unified segmentation. Neurolmage 2005, 26:839-851.

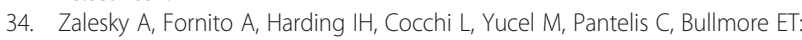
Whole-brain anatomical networks: does the choice of nodes matter? Neurolmage 2010, 50:970-983.

35. Tzourio-Mazoyer N, Landeau B, Papathanassiou D, Crivello F, Etard O, Delcroix N, Mazoyer B, Joliot M: Automated anatomical labeling of activations in SPM using a macroscopic anatomical parcellation of the MNI MRI single-subject brain. Neurolmage 2002, 15:273-289.

36. He Y, Chen ZJ, Evans AC: Small-world anatomical networks in the human brain revealed by cortical thickness from MRI. Cerebral cortex 2007 17:2407-2419.

37. van Wijk BC, Stam CJ, Daffertshofer A: Comparing brain networks of different size and connectivity density using graph theory. PLoS One 2010, 5:e13701.

38. Rubinov M, Sporns O: Complex network measures of brain connectivity: uses and interpretations. Neurolmage 2010, 52:1059-1069.

39. Maslov S, Sneppen K: Specificity and stability in topology of protein networks. Science 2002, 296:910-913.

40. Milo R, Shen-Orr S, Itzkovitz S, Kashtan N, Chklovskii D, Alon U: Network motifs: simple building blocks of complex networks. Science 2002, 298:824-827.

41. Supekar K, Menon V, Rubin D, Musen M, Greicius MD: Network analysis of intrinsic functional brain connectivity in Alzheimer's disease. PLOS Comput Biol 2008, 4:e1000100.
42. Sailer M, Fischl B, Salat D, Tempelmann C, Schoenfeld MA, Busa E, Bodammer $\mathrm{N}$, Heinze $\mathrm{HJ}$, Dale A: Focal thinning of the cerebral cortex in multiple sclerosis. Brain 2003, 126:1734-1744

43. Sepulcre J, Goni J, Masdeu JC, Bejarano B, Velez N, Toledo JB, Villoslada P: Contribution of white matter lesions to gray matter atrophy in multiple sclerosis. Arch Neurol 2009, 66:173-179.

44. Dietrich J, Han R, Yang Y, Mayer-Proschel M, Noble M: CNS progenitor cells and oligodendrocytes are targets of chemotherapeutic agents in vitro and in vivo. $J$ Biol 2006, 5:22

45. Seigers R, Schagen SB, Beerling W, Boogerd W, van Tellingen O, van Dam FS, Koolhaas JM, Buwalda B: Long-lasting suppression of hippocampal cell proliferation and impaired cognitive performance by methotrexate in the rat. Behav Brain Res 2008, 186:168-175.

46. Winocur G, Vardy J, Binns MA, Kerr L, Tannock I: The effects of the anticancer drugs, methotrexate and 5-fluorouracil, on cognitive function in mice. Pharmacol Biochem Behav 2006, 85:66-75.

47. Han R, Yang YM, Dietrich J, Luebke A: Systemic 5-fluorouracil treatment causes a syndrome of delayed myelin destruction in the central nervous system. J Biol 2008, 7(4):12

48. Seigers R, Schagen SB, Coppens CM, van der Most PJ, van Dam FS, Koolhaas JM, Buwalda B: Methotrexate decreases hippocampal cell proliferation and induces memory deficits in rats. Behav Brain Res 2009, 201:279-284.

49. Sporns O: The human connectome: a complex network. Annals NewYork Academy Sci 2011, 1224:109-25.

50. Kesler SR, Bennett FC, Mahaffey ML, Spiegel D: Regional brain activation during verbal declarative memory in metastatic breast cancer. Clin Cancer Res 2009, 15:6665-6673.

51. Silverman DH, Dy CJ, Castellon SA, Lai J, Pio BS, Abraham L, Waddell K, Petersen L, Phelps ME, Ganz PA: Altered frontocortical, cerebellar, and basal ganglia activity in adjuvant-treated breast cancer survivors 510 years after chemotherapy. Breast Cancer Res Treat 2007, 103:303-311.

52. Samatovicz RA: Genetics and brain injury: apolipoprotein E. J head trauma rehab 2000, 15:869-874.

53. Ahles TA, Saykin AJ, Noll WW, Furstenberg CT, Guerin S, Cole B, Mott LA: The relationship of APOE genotype to neuropsychological performance in long-term cancer survivors treated with standard dose chemotherapy. Psycho-Oncology 2003, 12:612-619.

54. Krabbe KS, Reichenberg A, Yirmiya R, Smed A, Pedersen BK, Bruunsgaard H: Low-dose endotoxemia and human neuropsychological functions. Brain Behav Immun 2005, 19:453-460.

55. Wilson CJ, Finch CE, Cohen HJ: Cytokines and cognition-the case for a head-to-toe inflammatory paradigm. J Am Geriatr Soc 2002, 50:2041-2056.

56. Aalto S, Bruck A, Laine M, Nagren K, Rinne JO: Frontal and temporal dopamine release during working memory and attention tasks in healthy humans: a positron emission tomography study using the highaffinity dopamine D2 receptor ligand [11 C]FLB 457. I neurosci official $j$ Soc Neurosci 2005, 25:2471-2477.

57. Vehmanen L, Elomaa I, Blomqvist C, Saarto T: Tamoxifen treatment after adjuvant chemotherapy has opposite effects on bone mineral density in premenopausal patients depending on menstrual status. J Clin Oncol 2006, 24:675-680

58. Boulware MI, Kent BA, Frick KM: The Impact of Age-Related Ovarian Hormone Loss on Cognitive and Neural Function. Curr Top Behav Neurosci 2012, 10:165-184.

59. Castellon SA, Ganz PA, Bower JE, Petersen L, Abraham L, Greendale GA: Neurocognitive performance in breast cancer survivors exposed to adjuvant chemotherapy and tamoxifen. J Clin Exp Neuropsychol 2004 26:955-969.

60. Eberling JL, Wu C, Tong-Turnbeaugh R, Jagust WJ: Estrogen- and tamoxifen-associated effects on brain structure and function. Neurolmage 2004, 21:364-371.

61. Poreh A: The Quantified Process Approach to Neuropsychological Assessment. New York, NY: Taylor and Francis; 2006.

doi:10.1186/1471-2377-12-28

Cite this article as: Hosseini et al:: Altered small-world properties of gray matter networks in breast cancer. BMC Neurology 2012 12:28. 